\section{Fatores associados à recorrência da gravidez na adolescência em uma maternidade escola: estudo caso-controle}

\author{
A case-control study of factors associated with \\ repeat teen pregnancy based on a sample \\ from a university maternity hospital
}

\author{
Factores asociados con la recurrencia de \\ embarazos de adolescentes en una maternidad \\ escuela: estudio de casos y controles
}

Andréa de Albuquerque Arruda Silva 1 Isabela C. Coutinho 1

Leila Katz ${ }^{1}$

Alex Sandro Rolland Souza 1

\footnotetext{
1 Instituto de Medicina Integral Professor Fernando Figueira, Recife, Brasil.

Correspondência I. C. Coutinho Instituto de Medicina Integral Professor Fernando Figueira. Rua dos Coelhos 300, Recife, PE 50070-550, Brasil. isabelacoutinhoneiva@gmail. com
}

\begin{abstract}
Repeat teen pregnancy is a frequent issue and is considered an aggravating factor for increased maternal and fetal morbidity and social problems. The aim of the study was to identify factors associated with repeat teen pregnancy. A case-control study was conducted in 90 postpartum adolescents with more than one pregnancy (cases) and 90 adult women with a history of only one pregnancy during adolescence (controls). Statistical analysis used hierarchical logistic regression with 5\% significance. Early sexual initiation (< 15 years), early age at first pregnancy ( $<16$ years), not raising the children themselves, and low family income $(<$ one minimum wage) were associated with repeat teenage pregnancy, while partner change was inversely associated. Repeat teen pregnancy was mainly associated with reproductive and socioeconomic factors. Partner change appeared as a protective factor. Measures should be adopted during the postpartum period of teenage mothers in order to avoid repeat pregnancy.
\end{abstract}

Pregnancy in Adolescence; Unplanned Pregnancy; Adolescent Health; Recurrence

\section{Resumo}

Recorrência da gravidez na adolescência é uma situação frequente, sendo considerada como um fator agravante tanto para o aumento da morbidade materna e fetal quanto para aumento de problemas sociais. O objetivo desta pesquisa foi identificar fatores associados à recorrência de gravidez em adolescentes. Realizou-se estudo caso-controle com puérperas, incluindo 90 adolescentes com mais de uma gravidez (caso) e 90 adultas, mulheres que tiveram uma gestação na adolescência, mas que não recorreram (controle). Para análise estatística, utilizou-se regressão logística hierarquizada, comníveldesignificância de 5\%. Os fatores que permaneceram associados à recorrência da gravidez na adolescência foram: coitarca $<15$ anos, idade da primeira gestação < 16 anos, mudança de parceiro, não cuidar dos filhos e renda familiar < um salário mínimo. A recorrência de gravidez na adolescência foi associada especialmente a fatores reprodutivos e socioeconômicos. A mudança de parceiro foi fator de proteção. No puerpério de adolescentes, devem ser intensificados os cuidados para que seja evitada a recorrência.

Gravidez na Adolescência; Gravidez não Planejada; Saúde na Adolescência; Recidiva 


\section{Introdução}

A Organização Mundial da Saúde (OMS) define a adolescência como o período compreendido entre os 10 e 19 anos 1 . Fase da vida entre a infância e a idade adulta, é marcada por um processo com sucessivas modificações de crescimento e de desenvolvimento biopsicossocial, em que o individuo se desenvolve física e emocionalmente, quando, muitas vezes, ocorre o início da vida sexual 2

Nos últimos anos, ocorreram muitas mudanças quanto ao comportamento dos indivíduos, particularmente no que se refere ao aumento da atividade sexual entre os adolescentes, levando a um aumento de gravidez muitas vezes não planejada ${ }^{3}$.

Inúmeras causas podem estar envolvidas com a ocorrência da gestação na adolescência, em especial a não planejada ou a indesejada. Entre essas, destacamos os fatores clínicos, sociais, culturais e emocionais. E como consequência ocorrem modificações no projeto de vida do adolescente, limitando ou adiando a possibilidade de engajamento dessas jovens na sociedade 4 .

A gestação em adolescentes encontra-se associada à baixa adesão ao pré-natal, o que pode ocasionar maior prevalência de recém-nascido de baixo peso, parto pré-termo e aumentar a necessidade de suporte psicossocial ocasionado pelo estresse da gravidez nessa fase da vida 5 .

A dificuldade de ter uma pessoa para ajudar a cuidar do filho, as modificações clínicas decorrentes da gravidez e a falta de interesse em frequentar as aulas, durante essa fase, podem resultar em abandono escolar e baixa escolaridade, e consequente redução da chance para entrada no mercado de trabalho 6 .

Ainda como consequência da baixa escolaridade da mãe adolescente é sugerida a inadequada educação sexual. As informações necessárias a uma boa educação sexual dependem do ambiente familiar e da escola, compondo uma dificuldade de diálogo sobre a sexualidade, com evidente prejuízo do entendimento sobre a importância de usar métodos contraceptivos adequados. Isso pode contribuir com uma nova gestação não planejada, ainda nessa fase de vida, além de suas resultantes. Estudos observaram que a cada três adolescentes, uma havia tido recorrência em até dois anos, após a primeira gravidez. Assim, algumas adolescentes, por vezes, apresentam outras gestações sem planejamento, ocorrendo em curtos intervalos de tempo 7,8,9.

A multiparidade na adolescência é uma situação cada vez mais frequente, sendo considerada como um fator agravante tanto para o aumento da morbidade materna e fetal, quanto para pro- blemas de aspectos sociais 10. Tal preocupação se torna mais relevante quando se constata que a cada nova gravidez ocorre a diminuição da probabilidade de a adolescente concluir os estudos, de ter um emprego estável e de ser economicamente autossuficiente ${ }^{11}$.

O baixo nível socioeconômico, a pouca escolaridade da mãe ou do responsável, o casamento, o desejo da primeira gravidez e o uso inadequado de métodos anticoncepcionais são alguns fatores relacionados à repetição da gravidez em adolescentes 12 .

Ainda são escassos os trabalhos que avaliam os fatores associados à recorrência da gestação entre as adolescentes 10,12. Diante da necessidade de buscar mais conhecimentos sobre a recorrência da gravidez nessa fase da vida, conduzimos este estudo com o objetivo de identificar os fatores associados à repetição da gravidez na adolescência.

\section{Métodos}

Realizou-se um estudo do tipo caso-controle, no período de fevereiro a agosto de 2011, no setor de alojamento conjunto do Instituto de Medicina Integral Professor Fernando Figueira (IMIP), localizado no Recife, Pernambuco, situado na Região Nordeste do Brasil. Ele é um hospital de referência terciário que atende a população do Sistema Único de Saúde (SUS) e onde são assistidos cerca de 6 mil partos por ano.

A amostra do estudo foi constituída de puérperas adolescentes, segundo a definição da OMS 1, que tiveram mais de um parto durante a adolescência (caso/recorrência) e puérperas adultas, que tiveram uma gestação na adolescência e outra(s) na idade adulta (controle/não recorrência). Diariamente, foram identificadas as puérperas candidatas a inclusão no estudo (casos/recorrência), por meio de consulta ao livro de procedimentos obstétricos utilizado no setor de pré-parto e bloco obstétrico. Para cada caso, recorreu-se ao mesmo livro buscando uma candidata a controle que tivesse o horário de parto mais próximo ao do caso. Caso alguma candidata a controle não aceitasse participar, recorria-se à próxima mulher elegível com horário de parto mais próximo.

Foram critérios de inclusão para os casos: ser adolescente e ter vivenciado mais de uma gestação. Para os controles, a mulher deveria ter vivenciado uma gestação na adolescência e ter vivenciando uma nova gestação somente agora na idade adulta.

Depois de identificadas as pacientes, aplicou-se uma lista de checagem com os critérios 
de inclusão e exclusão, confirmando tratar-se de casos ou controles. Em seguida, explicaram-se os objetivos da pesquisa, convidando as puérperas a participar do estudo mediante assinatura do Termo de Consentimento Livre e Esclarecido por elas ou por seus responsáveis legais.

Foram excluídas, em ambos os grupos, puérperas com história de gravidez atual resultante de estupro, as inimputáveis e adolescentes menores de 18 anos, sem responsável legal presente.

Não foram encontrados estudos caso-controle avaliando recorrência de gestação na adolescência. O tamanho da amostra foi calculado no programa OpenEpi (Dean AG, Sullivan KM, Soe MM. OpenEpi: Open Source Epidemiologic Statistics for Public Health, http://www.Open Epi.com), considerando nas mulheres que tiveram recorrência, uma frequência de não morar com os pais de $61,5 \%$ 12. Supondo que entre as mulheres que não têm recorrência essa frequência fosse $38,5 \%$, seriam necessárias 150 mulheres (sendo 75 em cada grupo) para demonstrar diferença entre os grupos, com um poder de $80 \%$ e um intervalo de 95\% de confiança (IC95\%). Esse número foi acrescido de $20 \%$ prevendo possíveis perdas, totalizando 180 entrevistadas, com a distribuição de 90 para cada grupo.

Durante o período do estudo houve 3.611 partos no serviço. Foram identificadas $773 \mathrm{mu}-$ lheres que preenchiam os critérios de inclusão para caso ou controle e, dentre elas, foram abordadas 200. Das 103 candidatas a caso, 13 foram excluídas; das 97 mulheres candidatas a controles, 07 foram excluídas. Todas as mulheres excluídas o foram por ser inimputáveis. Restaram 180 mulheres, distribuídas entre 90 casos e 90 controles (Figura 1).

Construiu-se um modelo teórico com as variáveis a serem estudadas: características biológicas (idade e cor das puérperas), sociodemográficas (escolaridade da puérpera da genitora e de seu companheiro, religião, estado civil, renda familiar e o fato de sua genitora ter sido mãe na adolescência), reprodutivas (idade de ocorrência da menarca, da coitarca e da primeira gestação e o tipo de parto) e de assistência à saúde (orientação sobre métodos anticoncepcionais e a sua utilização, acesso aos serviços de saúde e planejamento das gestações). Além disso, foram avaliadas as características sobre aspectos familiares em relação à gestação na adolescência (ter bom relacionamento com os pais, quem eram os cuidadores dos filhos e a aceitação das gestações pela família). O valor do salário mínimo considerado foi o vigente na época do estudo.

$\mathrm{Na}$ análise estatística, realizada no programa Epi Info 3.5.3 (Centers for Disease Control and Prevention, Atlanta, Estados Unidos), foram cal- culados as médias e desvios-padrão para as variáveis numéricas e a distribuição de frequência, para as variáveis categóricas. Realizou-se uma análise bivariada para verificar a existência de diferenças entre os grupos, utilizando os testes qui-quadrado de associação (Pearson) e exato de Fisher, quando pertinentes, com nível de 5\% de significância de 5\%. Para determinar a força de associação, foram calculados a odds ratio (OR) e o seu IC95\%.

Todas as variáveis avaliadas na análise bivariada foram incluídas na análise multivariada pela regressão logística múltipla stepwise, com base em um modelo teórico hierarquizado. As variáveis foram divididas em blocos, sendo os fatores mais distais (nível 1) que se associam de forma mais indireta (escolaridade do companheiro e da genitora da parturiente, genitora da mulher foi mãe adolescente, não ter religião e não aceitação da gestação pela família e cor/raça) do que os mediais (tipo de parto anterior, não planejamento da gestação anterior, escolaridade de puérpera, repetência e/ou abandono escolar, falta de acesso aos serviços de saúde na gestação anterior e/ou atual, relacionamento familiar ruim e não orientação quanto a métodos contraceptivos nível 2), seguidas pelas variáveis do nível 3, que são, por fim, as consideradas mais próximas do desfecho (não planejamento da gestação atual, não cuidar dos filhos, idade menor que 16 anos, idade da menarca, coitarca antes dos 15 anos, ser solteira, mudança de parceiro, não ter parceiro fixo, renda familiar menor que um salário mínimo, não usar métodos contraceptivos, não amamentar). Destacaram-se de cada bloco as variáveis que persistiram associadas ao desfecho inicialmente no nível de significância de $20 \%$, realizando-se nova regressão e selecionando-se as variáveis do bloco que persistiram associadas ao desfecho no nível de 5\% de significância. Em seguida, as variáveis resultantes de cada bloco foram utilizadas em uma nova regressão em que se selecionaram, para cálculo do risco ajustado de recorrência de gestação na adolescência, aquelas que persistiram significativamente associadas ao desfecho, tendo em conta o nível de $5 \%$.

O estudo foi submetido ao Comitê de Ética em Pesquisa em Seres Humanos do IMIP, tendo sido iniciado apenas após a sua aprovação, sob protocolo no 2.046-11 de 27 de janeiro de 2011 .

\section{Resultados}

A média de idade no grupo de casos foi de 17,9 anos, variando de 15 a 19 anos (desvio-padrão - DP de 1,1 ano). No grupo controle, a média de idade foi de 28,1 anos, variando de 20 a 42 anos 



(DP $=5,2$ anos). Em relação à cor da pele, não houve diferença entre os grupos (Tabela 1).

Quarenta e uma mulheres no grupo dos casos $(45,6 \%)$ versus 25 (27,8\%) do grupo controle referiram não ter nenhuma religião. Entre aquelas que apresentaram recorrência de gestação, a chance de não ter religião foi aproximadamente duas vezes maior (OR: 2,2; IC95\%: 1,2-4,0; $\mathrm{p}=$ 0,01) (Tabela 1).

Entre os casos, a média de anos estudados foi de 6,7 anos ( $\mathrm{DP}=2,7$ anos) enquanto no controle foi de 8,3 anos ( $D P=3,6$ anos) ( $p=0,0007)$. Quando delimitamos um ponto de corte de 7 anos de estudo, verificou-se esse achado em 50 casos
$(61,7 \%)$ versus $32(36,4 \%)$ controles, de forma que entre as que tiveram recorrência de gravidez na adolescência, a chance de terem estudado menos do que 7 anos, foi cerca de três vezes maior (OR: 2,8; IC95\%: 1,5-5,3; p = 0,0009) (Tabela 1).

Não foram encontradas diferenças entre os grupos quando se avaliaram o estado civil, a escolaridade da mãe da puérpera, abandono e repetência escolar (Tabela 1) e idade da menarca (Tabela 2).

Observou-se que a escolaridade média do companheiro das mulheres estudadas foi de 6,2 anos (DP $=3,9$ anos) no grupo dos casos e 7,9 anos (DP $=4,0$ anos) para o grupo controle $(\mathrm{p}=$ 
Características biológicas e sociodemográficas, de acordo com a recorrência ou não da gravidez na adolescência.

\begin{tabular}{|c|c|c|c|c|c|c|c|}
\hline \multirow[t]{2}{*}{ Característica } & \multicolumn{2}{|c|}{$\begin{array}{l}\text { Recorrência } \\
\qquad(N=90)\end{array}$} & \multicolumn{2}{|c|}{$\begin{array}{l}\text { Não recorrência } \\
\qquad(N=90)\end{array}$} & \multirow[t]{2}{*}{ OR } & \multirow[t]{2}{*}{ IC95\% } & \multirow[t]{2}{*}{$\begin{array}{l}\text { Valor } \\
\text { de } p \text { * }\end{array}$} \\
\hline & $\mathbf{n}$ & $\%$ & $\mathbf{n}$ & $\%$ & & & \\
\hline Cor negra & 46 & 51,1 & 44 & 48,9 & 1,30 & $0,73-2,34$ & 0,371 \\
\hline Cor não negra & 40 & 44,4 & 50 & 55,6 & & & \\
\hline Ser solteira & 30 & 33,3 & 22 & 24,4 & 1,54 & $0,80-2,96$ & 0,188 \\
\hline Não ser solteira & 60 & 66,7 & 68 & 75,6 & & & \\
\hline Sem religião & 41 & 45,6 & 25 & 27,8 & 2,17 & $1,16-4,04$ & 0,013 \\
\hline Ter religião & 49 & 54,4 & 65 & 72,2 & & & \\
\hline Escolaridade da puérpera $<7$ anos & 50 & 61,7 & 32 & 36,4 & 2,82 & $1,51-5,26$ & $<0,001$ \\
\hline Escolaridade da puérpera $>7$ anos & 31 & 38,3 & 56 & 63,6 & & & \\
\hline Escolaridade da mãe da puérpera $<3$ anos & 43 & 47,8 & 47 & 52,2 & 0,83 & $0,46-1,50$ & 0,550 \\
\hline Escolaridade da mãe da puérpera $>3$ anos & 47 & 52,2 & 43 & 47,8 & & & \\
\hline Renda familiar $\leq 1$ salário mínimo & 75 & 83,3 & 55 & 61,1 & 3,18 & $1,58-6,39$ & $<0,001$ \\
\hline Renda familiar $\geq 1$ salário mínimo & 15 & 16,7 & 35 & 38,9 & & & \\
\hline Escolaridade do companheiro $<7$ anos & 51 & 56,7 & 35 & 38,9 & 2,05 & $1,13-3,72$ & 0,016 \\
\hline Escolaridade do companheiro $>7$ anos & 39 & 43,3 & 55 & 61,1 & & & \\
\hline Abandono escolar & 64 & 71,9 & 59 & 65,6 & 1,34 & $0,71-2,55$ & 0,359 \\
\hline Não ter abandonado a escola & 25 & 28,1 & 31 & 34,4 & & & \\
\hline Repetência escolar & 59 & 65,6 & 46 & 51,1 & 1,82 & $0,99-3,31$ & 0,049 \\
\hline Não ter repetência escolar & 31 & 34,4 & 44 & 48,9 & & & \\
\hline
\end{tabular}

IC95\%: intervalo de 95\% de confiança; OR: odds ratio.

* Teste de qui-quadrado.

0,003). Da mesma forma, quando se delimitou um ponto de corte de 7 anos estudados pelos companheiros das mulheres envolvidas, entre aquelas que apresentaram recorrência de gestação na adolescência, a chance de os parceiros terem uma escolaridade menor que 7 anos foi duas vezes maior (OR: 2,0; IC95\%: 1,1-3,7; $p=0,016$ ) (Tabela 1).

Já quando se avaliou a renda familiar, o grupo da recorrência (adolescentes) teve uma média de $1,1$ salário mínimo ( $\mathrm{DP}=0,5)$, enquanto no grupo controle a média foi de $1,5(\mathrm{DP}=0,8)$, havendo uma diferença estatisticamente significante entre os dois grupos ( $\mathrm{p}=0,004)$. Quando se considerou o ponto de corte de um salário mínimo, verificou-se que, no grupo de casos, 75 (83,3\%) mulheres recebiam menos que um salário mínimo; no grupo controle, 55 (61,1\%) mulheres tinham renda inferior a essa. Dessa forma, entre as mulheres com recorrência de gravidez, a chance de a renda familiar ser menor ou igual a um salário mínimo foi cerca de três vezes maior (OR: 3,8; IC95\%: 1,6-6,4; p = 0,0008) (Tabela 1).

A idade média da coitarca foi de 13,9 anos (DP $=1,4)$, e nos controles a média encontrada foi de 15,7 anos (DP = 1,7) $(\mathrm{p}<0,0001)$. Quando se estabeleceu o ponto de corte de 15 anos para a ocorrência da coitarca, constatou-se que, entre os casos, 77 mulheres $(85,6 \%)$ do grupo de casos e $33(42,2 \%)$ dos controles tiveram a sua primeira relação sexual antes de 15 anos. Assim, entre os casos, a chance de ter havido coitarca antes dos 15 anos de idade foi cerca de oito vezes maior (OR: 8,1; IC95\%: 3,9-16,7; p < 0,0001) (Tabela 2).

Quanto à ocorrência da primeira gestação, a média de idade entre os casos foi de 15,2 anos (DP $=1,4$ ano) e no controle foi de 17,2 anos (DP = $1,5$ ano) ( $\mathrm{p}<0,0001)$. Ao se estabelecer um ponto de corte de 16 anos para a primeira gestação, 74 mulheres $(71,1 \%)$ do grupo de casos e $29(32,2 \%)$ do controle engravidaram antes dessa idade ( $\mathrm{p}<0,0001)$. Entre o grupo da recorrência, a chance de ter engravidado pela primeira vez antes de 16 anos foi cerca de cinco vezes maior (OR: 5,2; IC95\%: 2,7-9,8; p < 0,0001). Entre as mulheres que apresentaram recorrência de gravidez, houve diferença entre os grupos em relação a ter parceiro fixo e não ter amamentado após a última gravidez (Tabela 2).

Não se observou diferença estatisticamente significante entre os grupos em relação: ao tipo de parto anterior experimentado pelas mulheres 
Características reprodutivas e da assistência à saúde, de acordo com a recorrência ou não da gravidez na adolescência.

\begin{tabular}{|c|c|c|c|c|c|c|c|}
\hline \multirow[t]{2}{*}{ Característica } & \multicolumn{2}{|c|}{$\begin{array}{l}\text { Recorrência } \\
\qquad(N=90)\end{array}$} & \multicolumn{2}{|c|}{$\begin{array}{l}\text { Não recorrência } \\
\qquad(\mathrm{N}=90)\end{array}$} & \multirow[t]{2}{*}{ OR } & \multirow[t]{2}{*}{ IC95\% } & \multirow[t]{2}{*}{$\begin{array}{l}\text { Valor } \\
\text { de } p \text { * }\end{array}$} \\
\hline & $n$ & $\%$ & $\mathrm{n}$ & $\%$ & & & \\
\hline Coitarca $<15$ anos & 77 & 85,6 & 38 & 42,2 & 8,10 & $3,94-16,67$ & $<0,001$ \\
\hline Coitarca $>15$ anos & 13 & 14,4 & 52 & 57,8 & & & \\
\hline 1ạ gestação $\leq 16$ anos & 64 & 71,1 & 29 & 32,2 & 5,17 & $2,74-9,77$ & $<0,001$ \\
\hline 1a gestação $\geq 16$ anos & 26 & 28,9 & 61 & 67,8 & & & \\
\hline Cesariana anterior & 41 & 45,6 & 38 & 42,2 & 1,14 & $0,63-2,06$ & 0,652 \\
\hline Não houve cesárea na última gestação & 49 & 54,4 & 52 & 57,8 & & & \\
\hline Não ter parceiro fixo atual & 72 & 80,0 & 81 & 91,0 & 0,39 & $0,16-0,96$ & 0,036 \\
\hline Ter parceiro fixo & 18 & 20,0 & 8 & 9,0 & & & \\
\hline Mudança de parceiro & 44 & 48,9 & 53 & 58,9 & 0,66 & $0,37-1,20$ & 0,178 \\
\hline Não mudou de parceiro & 46 & 51,1 & 37 & 41,1 & & & \\
\hline $\begin{array}{l}\text { Não recebeu orientação sobre métodos } \\
\text { anticoncepcionais após a última gestação }\end{array}$ & 19 & 21,1 & 31 & 34,4 & 0,50 & $0,26-0,99$ & 0,045 \\
\hline $\begin{array}{l}\text { Recebeu orientação sobre métodos } \\
\text { anticoncepcionais após a última gestação }\end{array}$ & 71 & 78,9 & 59 & 65,6 & & & \\
\hline Não uso de métodos anticoncepcionais & 58 & 64,4 & 48 & 53,3 & 1,58 & $0,87-2,88$ & 0,129 \\
\hline Usou métodos anticoncepcionais & 32 & 35,6 & 42 & 46,7 & & & \\
\hline $\begin{array}{l}\text { Falta de acesso ao Serviço de Saúde antes } \\
\text { da 1a gestação }\end{array}$ & 9 & 10,0 & 4 & 4,4 & 2,38 & $0,70-8,06$ & 0,149 ** \\
\hline $\begin{array}{l}\text { Houve acesso ao Serviço de Saúde antes } \\
\text { da } 1 \underline{a} \text { gestação }\end{array}$ & 81 & 90,0 & 86 & 95,6 & & & \\
\hline $\begin{array}{l}\text { Falta de acesso aos Serviços de Saúde } \\
\text { após a última gestação }\end{array}$ & 13 & 14,4 & 10 & 11,1 & 1,35 & $0,55-3,26$ & 0,502 \\
\hline $\begin{array}{l}\text { Acesso aos Serviços de Saúde após } \\
\text { a última gestação }\end{array}$ & 77 & 85,6 & 80 & 88,9 & & & \\
\hline Não planejou a 1a gestação & 54 & 60,0 & 54 & 60,0 & 1,00 & $0,55-1,81$ & 1,000 \\
\hline Planejou a 1ạ gestação & 36 & 40,0 & 36 & 40,0 & & & \\
\hline Não planejou a atual gestação & 56 & 62,2 & 46 & 51,1 & 1,57 & $0,87-2,85$ & 0,132 \\
\hline Planejou a atual gestação & 34 & 37,8 & 44 & 48,9 & & & \\
\hline Não amamentou após última gestação & 17 & 18,9 & 7 & 7,8 & 2,76 & $1,08-7,03$ & 0,028 \\
\hline Amamentou após última gestação & 73 & 81,1 & 83 & 92,2 & & & \\
\hline
\end{tabular}

IC95\%: intervalo de 95\% de confiança; OR: odds ratio.

* Teste de qui-quadrado;

** Fisher.

envolvidas, à mudança do parceiro sexual, à falta de orientação sobre métodos anticoncepcionais após a última gestação, ao uso do método contraceptivo, à falta de acesso ao serviço de saúde antes da primeira e após a última gestação, ao não planejamento da primeira e da última gestação (Tabela 2).

Não houve diferença estatisticamente significante entre os grupos quanto à não aceitação pela família da primeira gravidez, não ter um bom relacionamento com os pais e a genitora da mulher avaliada ter sido mãe adolescente. Vale ressaltar, contudo, que no grupo dos casos, 23 mulheres $(25,6 \%)$ versus $6(6,7 \%)$ dos controles não cuidavam de seus filhos. Entre as mulheres que tiveram recorrência de gravidez na adolescência, a chance de não cuidarem dos filhos foi aproximadamente cinco vezes maior que nos controles (OR: 4,8; IC95\%: 1,8-12,5; $\mathrm{p}=0$ 0,0005) (Tabela 3).

Após a análise de regressão logística hierarquizada, as variáveis persistentes associadas à recorrência de gravidez na adolescência com efeito significativo foram: coitarca $\leq 15$ anos (OR: 5,0; 
IC95\%: 2,2-11,7; $\mathrm{p}=0,0002)$, não cuidar dos filhos (OR: 4,0; IC95\%: 1,4-11,7; $\mathrm{p}=0,01$ ), idade da primeira gestação $\leq 16$ anos (OR: 2,7; IC95\%: 1,3-5,9; $\mathrm{p}=0,01$ ), mudança de parceiro (OR: 0,4; IC95\%: $0,2-0,9 ; \mathrm{p}=0,03)$ e renda familiar $\leq 1$ salário mínimo (OR: 2,8; IC95\%: 1,2-6,4; p < 0,02) (Tabela 4).

\section{Discussão}

Observou-se que a recorrência de gravidez na adolescência foi associada a fatores reprodutivos e socioeconômicos, como: a ocorrência da coitarca antes dos 15 anos, a adolescente não ser a responsável pelos cuidados do filho da primeira gestação, a primeira gravidez ter ocorrido antes dos 16 anos e ter renda familiar menor que um salário mínimo. A mudança de parceiro foi um fator de proteção para a recorrência da gravidez na adolescência.

$\mathrm{Na}$ análise bivariada, tal variável não estava associada à chance de recorrência, sendo evidenciada apenas após a regressão logística. A provável explicação para a não associação na análise bivariada se deve à interferência de fatores de confusão, que foram eliminados com o tratamento metodológico.

Observada a média de idade do grupo de adolescentes, percebe-se uma concordância com outros estudos realizados, tanto no Brasil quanto em outros países 13,14,15. Em um estudo realizado no Rio de Janeiro, dentre 12.168 adolescentes estudadas, $13,5 \%$ apresentavam gravidez recorrente na segunda metade da adolescência (entre 15 e 19 anos) 14 e em outro, que avaliou fatores associados com recorrência da gestação

Tabela 3

Aspectos familiares, de acordo com a recorrência ou não da gravidez na adolescência.

\begin{tabular}{|c|c|c|c|c|c|c|c|}
\hline \multirow[t]{2}{*}{ Característica } & \multicolumn{2}{|c|}{$\begin{array}{l}\text { Recorrência } \\
\qquad(N=90)\end{array}$} & \multicolumn{2}{|c|}{$\begin{array}{c}\text { Não } \\
\text { recorrência } \\
(\mathrm{N}=90)\end{array}$} & \multirow[t]{2}{*}{ OR } & \multirow[t]{2}{*}{ IC95\% } & \multirow[t]{2}{*}{$\begin{array}{l}\text { Valor } \\
\text { de } p \text { * }\end{array}$} \\
\hline & $\mathbf{n}$ & $\%$ & $\mathbf{n}$ & $\%$ & & & \\
\hline Não aceitação pela família da primeira gestação & 12 & 13,3 & 14 & 15,6 & 0,83 & $0,36-1,92$ & 0,671 \\
\hline Família aceitou a primeira gestação & 14 & 15,6 & 76 & 84,4 & & & \\
\hline Não ter bom relacionamento com os pais & 14 & 15,6 & 12 & 13,3 & 1,19 & $0,52-2,75$ & 0,671 \\
\hline Tem bom relacionamento com os pais & 76 & 84,4 & 78 & 86,7 & & & \\
\hline Genitora foi mãe adolescente & 64 & 71,1 & 59 & 65,6 & 1,29 & $0,68-2,42$ & 0,423 \\
\hline Genitora não foi mãe adolescente & 26 & 28,9 & 31 & 34,4 & & & \\
\hline Não cuida dos filhos & 23 & 25,6 & 6 & 6,7 & 4,80 & $1,85-12,47$ & $<0,001$ \\
\hline Cuida dos filhos & 67 & 74,4 & 84 & 93,3 & & & \\
\hline
\end{tabular}

IC95\%: intervalo de 95\% de confiança; OR: odds ratio.

* Teste de qui-quadrado.

Tabela 4

Fatores associados à recorrência de gravidez na adolescência após análise multivariada.

\begin{tabular}{|c|c|c|c|c|c|}
\hline Variáveis & Coeficiente & Erro-padrão & OR & IC95\% & Valor de $p$ \\
\hline Coitarca $\leq 15$ anos & 1,62 & 0,43 & 5,0 & $2,2-11,7$ & 0,0002 \\
\hline Não cuida dos filhos & 1,39 & 0,55 & 4,0 & $1,4-11,7$ & 0,01 \\
\hline Idade da 1 ạ gestação $\leq 16$ anos & 1,00 & 0,39 & 2,7 & $1,3-5,9$ & 0,01 \\
\hline Mudança de parceiro & $-0,81$ & 0,38 & 0,4 & $0,2-0,9$ & 0,03 \\
\hline Renda familiar $\leq 1 \mathrm{SM}$ & 1,02 & 0,43 & 2,8 & $1,2-6,4$ & 0,02 \\
\hline Constante & $-2,11$ & 0,50 & * & * & $<0,0001$ \\
\hline
\end{tabular}

IC95\%: intervalo de 95\% de confiança; OR: odds ratio; SM: salário mínimo. 
em curto período de tempo, a média de idade foi 16,7 anos 15. A provável explicação para esses estudos terem encontrado a gravidez na segunda metade da adolescência deve ser pela natureza dos estudos (de avaliação da recorrência num curto período de tempo).

Idade da coitarca inferior a 15 anos, ter engravidado pela primeira vez antes dos 16 anos e não amamentar após a última gestação foram as características reprodutivas e de assistência à saúde que se associaram a uma maior chance de recorrência de gravidez na adolescência. Em relação aos aspectos familiares, apenas o fato de não cuidar dos filhos das gestações anteriores se associou a uma maior chance de gestação recorrente nessa fase da vida.

Após a análise de regressão logística múltipla, verificou-se que entre as mulheres que apresentaram gestação recorrente na adolescência a chance de ter vivenciado a coitarca antes dos 15 anos foi cerca de cinco vezes maior. Outro estudo, realizado no Nordeste do Brasil 16, descreve gestações ocorrendo cedo durante a adolescência, o que pode ser explicado pelas características de vida dessas duas populações. Em relação à recorrência, poder-se-ia inferir que o fato de a gestação ter ocorrido de forma tão precoce, implica um maior período de exposição até o final da adolescência, aumentando a probabilidade de ocorrer nova gestação.

Outros estudos, realizados na Austrália e nos Estados Unidos, não confirmaram esse achado de que a idade da primeira relação sexual interfira com a chance de recorrência de gestação 11,15. Possivelmente, a diferença de fatores socioeconômicos e culturais, além da diferente disponibilidade e acesso a serviços de saúde em países desenvolvidos possam explicar essa distinção de resultados. É importante destacar que os estudos brasileiros foram realizados em hospitais públicos que concentram atendimento para uma clientela socialmente desfavorecida. Seria importante realizar estudos analisando adolescentes de diferentes estratos para verificar se esse achado pode ser extrapolado para todas as adolescentes de nossa região.

Observou-se que entre as adolescentes com gestação recorrente a chance de a primeira gestação ter acontecido antes dos 16 anos foi aproximadamente três vezes maior. A idade na primeira gestação já caracteriza que não houve cuidados preventivos e quanto mais cedo e mais imatura a adolescente, menor a probabilidade de mudança desse comportamento. Nos estudos brasileiros, essa variável específica não foi estudada, porém, novamente, o estudo australiano não encontrou que a idade precoce no momento da primeira gravidez tenha influenciado o risco de recorrên- cia 11. Entretanto, um estudo americano encontrou que engravidar antes dos quinze anos protegeu a adolescente de uma rápida repetição da gestação (dentro de 24 meses) 17.

Entre mulheres que tiveram recorrência de gestação ainda na adolescência, ocorreu uma chance quatro vezes maior de não cuidar da prole da gestação anterior, deixando a mulher mais livre, aumentando sua susceptibilidade de engravidar novamente. Ela poderia acreditar que, uma vez mais, contaria com outras pessoas para assumirem o outro filho, visto que uma adolescente que arca com os cuidados e responsabilidades de seu filho, provavelmente amadurece e é confrontada com as consequências de seus atos. Acreditamos que isso a protegeria de uma nova gestação. Além do que, no momento em que alguém assumiu os cuidados da prole anterior, presume-se que a receptividade da criança pela família foi boa, deixando a adolescente em uma situação confortável e segura de que, em se repetindo uma nova gravidez, seria novamente acolhida.

A situação econômica da família da adolescente, traduzida pela renda familiar, foi descrita por outros autores como um fator importante para ocorrência de gestação durante a adolescência. Alguns autores atentam para o fato de que a gravidez seja perpetuadora da baixa condição socioeconômica 18,19. Este estudo encontrou que, entre as mulheres que apresentaram recorrência de gestação na adolescência, houve uma chance quase três vezes maior quando a renda familiar era inferior a um salário mínimo. Contudo, outros autores não encontraram resultado semelhante, em população semelhante a nossa, quanto à baixa renda familiar 16. Cabe citar aqui que outro estudo, apesar de não ter avaliado a renda familiar média, ou outras características como baixa escolaridade e ser atendida pelo sistema público de saúde, destaca tais fatores como indicadores de baixa renda. Essas adolescentes foram estudadas e associadas ao risco aumentado de recorrência 12 .

$\mathrm{Na}$ análise bivariada, variáveis como baixa escolaridade da puérpera e de seu companheiro e repetência escolar - que podem refletir uma condição de baixa renda - estiveram associados a uma maior chance de recorrência. É possível que esses fatores todos estejam intrincados, justificando que na análise de regressão logística a baixa renda, fator preponderante e mais associado ao desfecho, tenha se sobressaído. Por outro lado, em relação à escolaridade da puérpera e do seu companheiro é possível que ela seja maior nos controles, pelo fato de esse grupo ter mais idade.

O relacionamento estável com o pai da criança aumenta o risco de uma nova gestação entre 
as adolescentes 20,21. Nossos dados apontam que a troca de parceiro diminui a chance de recorrência de gestação na adolescência em 56\%. É possível que a adolescente, ao sentir-se confortável e segura num relacionamento estável, adote cuidados contraceptivos menos rigorosos do que adotaria em caso de troca de parceiro.

Por outro lado, foi observado em um estudo americano que não estar com o pai da criança três meses após o parto foi preditor de recorrência de gestação nesse grupo 22. Esse achado foi semelhante a um estudo realizado em Fortaleza (Ceará), que encontrou um aumento de $40 \%$ no risco de recorrência quando houve troca de parceiro 12 . Isso poderia ser explicado pelo fato de o novo parceiro desejar também um filho com a companheira. No entanto, em estudo australiano, não foi percebida nenhuma influência do relacionamento com o pai da criança e a chance de recorrência da gravidez ${ }^{11}$. Esse fator precisa ser mais bem avaliado em futuros estudos.

Como limitações deste estudo, ressaltam-se os vieses decorrentes do próprio desenho de estudo, como vieses de seleção e de memória. Como o estudo teve base hospitalar, é possível que adolescentes atendidas em nosso serviço sejam diferentes de adolescentes atendidas em maternidades de baixo risco ou em outras regiões do Brasil. Por isso, não é recomendável a extrapolação dos resultados obtidos para toda a população de adolescentes.

Além disso, o próprio método de coleta pode ter acarretado imprecisão de algumas respostas, porque as entrevistas das adolescentes eram, na maioria das vezes, realizadas na presença de seu acompanhante. Não se acredita, todavia, que esse viés comprometeu o resultado, pois as variáveis que se associaram na análise não são variáveis que poderiam ser modificadas na presença de um acompanhante.

Diante dos resultados obtidos no presente estudo, é importante enfatizar que a adolescência é um período que ainda carece de especial atenção por parte dos serviços de saúde, apesar de existirem programas destinados a esse público. Programas de monitorização de adolescentes devem ser intensificados com o objetivo de prevenir gestações não desejadas ou não planejadas nessa fase da vida.

Nas situações em que ocorre uma gestação sem planejamento, os fatores de risco para sua recorrência devem ser reconhecidos de pronto, e os cuidados preventivos para uma nova gestação devem ser trabalhados com a adolescente desde o seu pré-natal.

Antes da alta hospitalar, quando da ocasião do nascimento do primeiro filho, deve ser realizada uma intervenção com o intuito de estimular a adoção de métodos contraceptivos, de modo a não deixar a puérpera adolescente sair sem optar pelo método que será utilizado para a sua contracepção. Consultas e busca ativa, quando necessárias, devem ser realizadas em ambulatórios de pós-natal, onde profissionais de saúde deveriam prestar assistência integral à adolescente, com o apoio de psicólogos, assistentes sociais, médicos (tocoginecologistas e pediatras) e enfermeiros, com experiência e disponibilidade a fim de prevenir uma nova gravidez.

\section{Resumen}

La recurrencia del embarazo en la adolescencia es una situación frecuente, siendo considerada como un factor agravante, tanto para el aumento de la morbilidad materna y fetal, como para el aumento de problemas sociales. El objetivo de esta investigación fue identificar factores asociados a la recurrencia de embarazo en adolescentes. Se realizó un estudio de caso-control con puérperas, incluyendo 90 adolescentes con más de un embarazo (caso) y 90 adultas, mujeres que tuvieron una gestación en la adolescencia, pero que no recurrieron (control). Para el análisis estadístico, se utilizó la regresión logística jerarquizada, con un nivel de significancia de un 5\%. Los factores que permanecieron asociados a la recurrencia de embarazo en la adolescencia fueron: coitarca < 15 años, edad de la primera gestación < 16 años, cambio de pareja, no cuidar de los hijos y renta familiar < un salario mínimo. La recurrencia de embarazo en la adolescencia fue asociada especialmente a factores reproductivos y socioeconómicos. El cambio de pareja fue un factor de protección. En el puerperio de adolescentes, se deben intensificar los cuidados para que se evite la recurrencia.

Embarazo en Adolescencia; Embarazo no Planeado; Salud del Adolescente; Recurrencia 


\section{Colaboradores}

A. A. A. Silva participou da concepção e desenho do estudo, realizou a coleta de dados, além da análise e interpretação dos dados e elaboração do manuscrito. I. C. Coutinho, L. Katz e A. S. R. Souza participaram da concepção e desenho do estudo, da análise e interpretação dos dados e elaboração do manuscrito.

\section{Referências}

1. World Health Organization. Young people's health: a challenge for society. Report of a WHO Study Group on Young People and Health for All by the Year 2000. Geneva: World Health Organization; 1986.

2. Nery IS, Mendonça RCM, Gomes IS, Fernandes ACN, Oliveira DC. Reincidência de gravidez em adolescentes de Teresina, PI, Brasil. Rev Bras Enferm 2011; 64:31-7.

3. Gradim CVC, Ferreira MBL, Moraes MJ. O perfil das grávidas adolescentes em uma unidade de saúde da família de Minas Gerais. Revista APS 2010; 13:55-61.

4. Cabral CS. Contracepção e gravidez na adolescência na perspectiva de jovens de uma comunidade favelada do Rio de Janeiro. Cad Saúde Pública 2003; 19:283-92.

5. Martins MG, Santos GHN, Sousa MS, Costa JEFB, Simões VMF. Associação de gravidez na adolescência e prematuridade. Rev Bras Ginecol Obstet 2011; 33:354-60.

6. Santos JO, Silva CFS, Petenão E, Soster FCB, Berard MB, Silva SR. Perfil das adolescentes com reincidência de gravidez assistidas no setor público de Indaiatuba (SP). Rev Inst Ciênc Saúde 2009; 27:115-21
7. Sousa MCR, Gomes KRO. Conhecimento objetivo e percebido sobre contraceptivos hormonais orais entre adolescentes com antecedentes gestacionais. Cad Saúde Pública 2009; 25:645-54.

8. Rosa AJ, Reis AOA, Tanaka ACA. Gestações sucessivas na adolescência. Rev Bras Crescimento Desenvolv Hum 2007; 17:165-72.

9. Persona L, Shimo AKK, Tarallo MC. Perfil de adolescentes com repetição da gravidez atendidas num ambulatório de pré-natal. Rev Latinoam Enferm 2004; 12:745-50.

10. Samandari G, Speizer IS. Adolescent sexual behavior and reproductive outcomes in Central America: trends over the past two decades. Int Perspect Sex Reprod Health 2010; 36:26-35.

11. Lewis LN, Doherty DA, Hickey M, Skinner SR. Predictors of sexual intercourse and rapid-repeat pregnancy among teenage mothers: an Australian prospective longitudinal study. Med J Aust 2010; 193:338-42.

12. Bruno ZV, Feitosa FEDL, Silveira KP, Morais IQD, Bezerra MDF. Reincidência de gravidez em adolescentes. Rev Bras Ginecol Obstet 2009; 31:480-4. 
13. World Health Organization. Adolescent pregnancy: issues in adolescent health and development. http://whqlibdoc.who.int/publications / 2004 (acessado em 05/Jun/2011).

14. Silva KS, Rozenberg R, Bonan C, Chuva VCC, Costa SF, Gomes MASM. Gravidez recorrente na adolescência e vulnerabilidade social no Rio de Janeiro (RJ, Brasil): uma análise de dados do sistema de nascidos vivos. Ciênc Saúde Coletiva 2011; 16:2485-93.

15. Crittenden CP, Boris NW, Rice JC, Taylor CA, Olds DL. The role of mental health factors, behavioral factors, and past experiences in the prediction of rapid repeat pregnancy in adolescence. J Adolesc Health 2009; 44:25-32.

16. Amorim MMR, Lima LA, Lopes CV, Araújo DKL, Silva JGG, César LC, et al. Fatores de risco para gravidez na adolescência em uma maternidadeescola da Paraíba: estudo caso-controle. Rev Bras Ginecol Obstet 2009; 31:404-10.

17. Boardman LA, Allsworth J, Phipps MG, Lapane KL. Risk factors for unintended versus intended rapid repeat pregnancies among adolescents. J Adolesc Health 2006; 39:597-608.
18. Hobcraft J, Kiernan K. Childhood poverty, early motherhood and adult social exclusion. Br J Sociol 2001; 52:495-517.

19. Duarte CM, Nascimento VB, Akerman M. Adolescent pregnancy and social exclusion: analysis of intra-urban disparities. Rev Panam Salud Pública 2006; 19:236-43.

20. Gispert M, Brinich P, Wheeler K, Krieger L. Predictors of repeat pregnancies among low-income adolescents. Hosp Community Psychiatry 1984; 35:719-23.

21. Pfitzner MA, Hoff C, McElligott K. Predictors of repeat pregnancy in a program for pregnant teens. J Pediatr Adolesc Gynecol 2003; 16:77-81.

22. Raneri LG, Wiemann CM. Social ecological predictors of repeat adolescent pregnancy. Perspect Sex Reprod Health 2007; 39:39-47.

Recebido em 18/Jun/2012

Versão final reapresentada em 30/Out/2012

Aprovado em 22/Nov/2012 